\title{
On the squares of Weber's parabolic cylinder functions and certain integrals connected with them
}

\author{
By S. C. Mitra.
}

(Received 13th April, 1933; and in revised form 31st July, 1933. Read 5th May, 1933.

1. The parabolic cylinder functions $D_{n}(x)$ and $D_{-(n+1)}( \pm i x)$ are defined by ${ }^{1}$

$$
\begin{aligned}
D_{n}(x)= & \frac{\Gamma\left(\frac{1}{2}\right)}{\Gamma\left(\frac{1}{2}-\frac{1}{2} n\right)} 2^{\frac{1}{n} n} e^{-\frac{1}{2 x}{ }_{1} F_{1}\left\{-\frac{1}{2} n ; \frac{1}{2} ; \frac{1}{2} x^{2}\right\}} \\
& +\frac{\Gamma\left(-\frac{1}{2}\right)}{\Gamma\left(-\frac{1}{2} n\right)} 2^{\frac{1}{2} n-\frac{1}{2}} x e^{-\nmid x^{2}}{ }_{1} F_{1}\left\{\frac{1}{2}-\frac{1}{2} n ; ? ; \frac{1}{2} x^{2}\right\},
\end{aligned}
$$

for all values of $n$ and $x$.

They satisfy the differential equation

$$
\frac{d^{2} y}{d x^{2}}+\left(n+\frac{1}{2}-\frac{1}{4} x^{2}\right) y=0 .
$$

The recurrence formulae for $D_{n}(x)$ are

and

$$
D_{n+1}(x)-x D_{n}(x)+n D_{n-1}(x)=0,
$$

$$
D^{\prime}{ }_{n}(x)+\frac{1}{2} x D_{n}(x)-n D_{n-1}(x)=0 .
$$

The relation connecting $D_{n}(x)$ and $D_{-(n+1)}( \pm i x)$ is

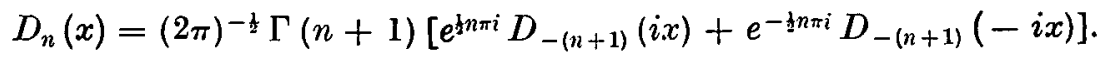

Let

$U_{n}(x)=e^{3 x^{2}} D_{n}(x)$.

When $n$ is a positive integer,

$U_{n}(x)=\left\{x^{n}-\frac{n(n-1)}{2} x^{n-2}+\frac{n(n-1)(n-2)(n-3)}{2.4} x^{n-4}-\ldots\right\}$,

the last term being $(-1)^{\frac{1}{2}(n-1)} \frac{n(n-1) \ldots 2}{2.4 \ldots(n-1)} x$ or $(-1)^{\frac{3}{2} n} \frac{n(n-1) \ldots 2.1}{2.4 \ldots n}$, according as $n$ is odd or even.

1 Whittaker and Watson, Modern Analysis (third edition), 347-49. 
This polynomial satisfies the recurrence formula

$$
U^{\prime}{ }_{n}(x)=n U_{n-1}(x),
$$

and is connected with the Hermite Polynomial by means of the relation

$$
U_{n}(x)=\frac{1}{2^{\frac{1}{2} n}} H_{n}\left(\frac{x}{\sqrt{2}}\right)
$$

2. Let

$$
y=D_{n}^{2}(x) .
$$

Then $D_{n}^{2}(x)$ satisfies the differential equation

$$
\frac{d^{3} y}{d x^{3}}+\left(4 n+2-x^{2}\right) \frac{d y}{d x}-x y=0
$$

Solutions ${ }^{1}$ of $(10)$ are $D_{n}^{2}(x), D_{-(n+1)}^{2}(i x), D_{-(n+1)}^{2}(-i x)$ and also the product of any two of $D_{n}(x), D_{-(n+1)}(i x), D_{-(n+1)}(-i x)$. The only solution which is odd in $x$ is $A\left[D_{-(n+1)}^{2}(i x)-D_{-(n+1)}^{2}(-i x)\right]$.

To solve the differential equation, let us write

$$
y=\Sigma a_{r} U_{r}(x) \text {. }
$$

Substituting in (10) we get after considerable simplification

$$
\Sigma a_{r}\left\{r(4 n+2-2 r) U_{r-1}(x)-(r+1) U_{r+1}(x)\right\}=0
$$

The three solutions are therefore

$y_{1}=a_{2 n+1}\left\{U_{2 n+1}(x)-\frac{(n+1)}{2(2 n+3)} U_{2 n+3}(x)+\frac{(n+1)(n+2)}{2.4(2 n+3)(2 n+5)} U_{2 n+5}(x)-\ldots\right\}$,

$y_{2}=b_{0}\left\{U_{0}(x)+\frac{1}{2(2 n-1)} \frac{1}{2} U_{2}(x)+\frac{1.3}{2.4(2 n-1)(2 n-3)} \frac{1}{2^{2}} U_{4}(x)+\ldots\right\}$

and

$y_{3}=c_{-1}\left\{U_{-1}(x)+\frac{1}{2}(4 n+4) U_{-3}(x)+\frac{1.3}{2.4}(4 n+4)(4 n+8) U_{-5}(x)+\ldots\right\}$.

1 Appell, Comptes Rendus, 91. The method had been previously used by Heine in a similar problem relating to $P_{m}(x) P_{n}(x)$. See the Appendix to Heine's Theorie der Kugelfunktionen (1878 edition).

My attention to this reference was drawn by a referee who kindly suggested some improvements also. 
The series (A) and (B) may, without difficulty, be shown to be absolutely and uniformly convergent in any finite interval $a \leqq x \leqq b$, by the aid of Watson's asymptotic formula ${ }^{1}$

$$
D_{-(n+1)}(-i z)=\frac{\sqrt{\pi}(\sqrt{n})^{n} e^{-t n}}{\Gamma(n+1)} e^{1 z^{2}} e^{i z^{\sqrt{n}}}\left[e^{-1 z^{2}}+\theta\right],
$$

where

and

$$
|\theta|<\frac{1}{|x|}\left[2 e^{-\frac{\pi}{3} n}\left(\frac{2}{\pi}\right)^{\frac{b}{b}}+8 A\left(\frac{2}{\pi n}\right)^{\frac{t}{2}}\left(1+e^{|y| k, N n}\right)\right]
$$

where

$$
|\theta|<\frac{1}{\sqrt{ } \mathbf{2}} e^{-3 n}+\left(\frac{2}{\pi}\right)^{\frac{1}{3}}\left\{k A+e^{-\frac{1}{3} n k^{2}} \sqrt{ } \pi\right\}\left\{1+e^{|y| k \sqrt{ } n}\right\},
$$

$$
z=x+i y, \quad k=2 n^{a-\frac{1}{t}}, \quad n>\exp \left\{\frac{2}{1-\frac{2}{2^{a}}} \log 2\right\}, \quad A=0 \cdot 742 \ldots \ldots,
$$

$|y|<\frac{1}{3} n^{\alpha}$ and $0 \leqq a<\frac{1}{2}$.

The series (C) is divergent and may be rejected.

Suppose $n$ is a positive integer. The series (A) and (B) give the expansions of $\left\{e^{(n+3) \pi i} D_{-(n+1)}^{2}(i x)+e^{-(n+i) \pi i} D_{-(n+1)}^{2}(-i x)\right\}$ and $D_{n}^{2}(x)$ respectively. Equating the coefficients of the general terms, we have

$$
\begin{aligned}
\left(e^{(n+1) \pi i} D_{-(n+1)}^{2}(i x)+e^{-(n+1) \pi i} D_{-(n+1)}^{2}(-i x)\right) & \\
& =\frac{\sqrt{2 \pi}}{\Gamma(2 n+2)}\left\{U_{2 n+1}(x)-\frac{(n+1)}{2(2 n+3)} U_{2 n+3}(x)\right. \\
& \left.+\frac{(n+1)(n+2)}{2.4(2 n+3)(2 n+5)} U_{2 n+5}(x)-\ldots\right\},
\end{aligned}
$$

and

$$
\begin{aligned}
D_{n}^{2}(x)= & \frac{1.3 \ldots(2 n-1)}{2^{n+\frac{1}{2}}}\left\{U_{0}(x)+\frac{1}{2(2 n-1)} \frac{1}{2} U_{2}(x)\right. \\
& \left.+\frac{1.3}{2.4(2 n-1)(2 n-3)} \frac{1}{2^{2}} U_{4}(x)+\ldots\right\} .
\end{aligned}
$$

These results can also be written in the form

$$
\begin{aligned}
& D_{0}(x)\left(e^{(n+1) \pi i} D_{-(n+1)}^{2}(i x)+e^{-(n+1) \pi i} D_{-(n+1)}^{2}(-i x)\right) \\
& =\frac{\sqrt{2 \pi}}{\Gamma(2 n+2)} \sum_{s=0}^{\infty}(-1)^{s} \frac{(n+1)(n+2) \ldots(n+s)}{2.4 \ldots 2 s(2 n+3)(2 n+5) \ldots(2 n+2 s+1)} D_{2 n+2 s+1}(x),
\end{aligned}
$$

${ }_{1}^{1}$ Proc. London Math. Soc. (2), 17 (1919), 116-48, 8 (1910), 416. See also a paper by Adamoft in Ann. de l'Institut Polytechnique de St Petersbourg, 5 (1906), 127-43. 
and

$D_{0}(x) D_{n}^{2}(x)$

$=\frac{1.3 \ldots(2 n-1)}{2^{n+\frac{1}{b}}} \sum_{s=0}^{\infty} \frac{1.3 \ldots(2 s-1)}{2.4 \ldots 2 s(2 n-1)(2 n-3) \ldots(2 n-2 s+1) 2^{s}} \times D_{2 s}(x)$.

It may be remarked here that (F) and (G) are also true when $n$ is zero, and (F) holds good for all positive values of $n$.

3. We might as well have assumed that

$$
y=e^{\frac{1}{2} x^{2}} \Sigma a_{-r} D_{-r}(i x),
$$

and tried to obtain a solution of the differential equation (10).

After some calculations, it is found that

$$
D_{0}(x) D_{-(n+1)}^{2}(i x)=\sum_{s=0}^{\infty} \frac{(n+1)^{2}(n+2)^{2} \ldots(n+s)^{2}}{s !} D_{-(2 n+2 s+2)}(i x) .
$$

The series is absolutely convergent and is true for all positive values of $n$ and also when $n$ is zero.

4. We shall now evaluate a few definite integrals.

Goldstein $^{1}$ has proved that when $a>0$ and $n$ is a positive integer

$\int_{0}^{\infty} x e^{\left(\frac{1}{x}-a\right) x^{n}} D_{2 n+1}(x) d x=\left(\frac{1}{2} \pi\right)^{\frac{1}{4}} \frac{\Gamma(2 n+2)}{\Gamma(n+1) 2^{n}}(2 \alpha)^{-\geqq}\left(\frac{1}{2} \alpha-1\right)^{n}$.

Consider the integral

$\int_{-\infty}^{\infty} x e^{-\alpha x z}\left(e^{\left(n+\frac{1}{2}\right) \pi i} D_{-(n+1)}^{2}(i x)+e^{-\left(n+\frac{1}{2}\right) \pi i} D_{-(n+1)}^{2}(-i x)\right) d x$.

$$
\text { ( } n=\mathbf{a} \text { positive integer). }
$$

By taking $a>0$, convergence at infinity is easily established.

Replacing the expression within the first bracket by the series (F), and integrating term by term with the help of the relation (13), we find that the integral is equal to

$$
\frac{4 \pi}{\Gamma(n+1)}(2 a)^{-3}\left(\frac{1}{2 a}-1\right)^{n}\left(\frac{1}{2 a}+1\right)^{-n-1}, \quad\left(\alpha>\frac{1}{6}\right) .
$$

1 Proc. London Math. Soc. (2), 34 (1932), 118. See also (2), 8 (1908), 397-400. 
The final series converges absolutely when $a>\frac{1}{6}$; and term by term integration is justified. ${ }^{1}$

\section{Therefore}

$$
\begin{aligned}
& \int_{-\infty}^{\infty} x e^{-a x^{2}}\left(e^{(n+1) \pi i} D_{-(n+1)}^{2}(i x)+e^{-(n+1) \pi i} D_{-(n+1)}^{2}(-i x)\right) d x \\
& =\frac{4 \pi}{\Gamma(n+1)}(2 \alpha)^{-3}\left(\frac{1}{2 \alpha}-1\right)^{n}\left(\frac{1}{2 \alpha}+1\right)^{-n-1}, \quad\left(a>\frac{1}{b}\right) .
\end{aligned}
$$

From $(\mathbf{E})$ we get the following results,

$$
\begin{aligned}
& \int_{0}^{\infty} e^{-3 x^{2}} D_{n}^{2}(x) D_{2 p}(x) d x \\
& =\sqrt{ } \pi \frac{\{1.3 \ldots(2 n-1)\}\{1.3 \ldots(2 p-1)\} \Gamma(2 p+1)}{2.4 \ldots 2 p(2 n-1)(2 n-3) \ldots(2 n-2 p+1) 2^{n+p+1}} ;(p \geqq 1) \\
& =\sqrt{ } \pi \frac{1.3 \ldots(2 n-1)}{2^{n+1}}, \quad(p=0) . \\
& \int_{0}^{z} D_{n}^{2}(z) d z=\frac{1.3 \ldots(2 n-1)}{2^{n+1}} e^{t z^{2}} \times \\
& \sum_{s=0}^{\infty} \frac{1.3 \ldots(2 s-1)}{2.4 \ldots 2 s(2 n-1)(2 n-3) \ldots(2 n-2 s+1) 2^{s}} \frac{D_{2 s+1}(z)}{2 s+1}
\end{aligned}
$$

Finally we have the integral

$$
\begin{aligned}
& \int_{0}^{\infty} D_{n}^{2}(x+t) d x=\left(\frac{1}{2} \pi\right)^{\frac{1}{2}} \Gamma(n+1)-\frac{1.3 \ldots(2 n-1)}{2^{n+\frac{t}{2}}} e^{t t^{2}} \times \\
& \sum_{s=0}^{\infty} \frac{1.3 \ldots(2 s-1)}{2.4 \ldots 2 s(2 n-1)(2 n-3) \ldots(2 n-2 s+1) 2^{s}} \frac{D_{2 s+1}(t)}{2 s+1} .
\end{aligned}
$$

5. The series $(G)$ can also be obtained in another way. Goldstein ${ }^{2}$ has shown that when $u$ is positive

$$
u^{-\mu} e^{-i y^{2} / u^{2}} D_{\mu-1}\left(\frac{y}{u}\right)=e^{-1 y^{2}} \sum_{s=0}^{\infty} \frac{\left(u^{2}-1\right)^{8}}{2^{8} \cdot s !} D_{\mu+2 s-1}(y) .
$$

Putting $\mu=2 n+1$ and $u=\frac{1}{\sqrt{ } 2}$, we get

$$
2^{n+1} D_{2 n}(\sqrt{2} y)=\sum_{s=0}^{\infty}(-1)^{s} \frac{1}{2^{2 s} s !} U_{2 n+2 s}(y) .
$$

${ }^{1}$ Cf. Bromwich, Theory of Infinite Series. $\$ 176$.

${ }^{2}$ loc, eit., 116. 
We have shown elsewhere ${ }^{1}$ that, when $n$ is a positive integer,

$D_{n}^{2}(x)=\frac{1}{2^{n}}\left\{D_{2 n}(x \sqrt{ } 2)+\frac{1 . n}{1 !} D_{2 n-2}(x \sqrt{ } 2)+\frac{1.3 n(n-1)}{2 !} D_{2 n-u}(x \sqrt{ } 2)\right.$

$\left.+\ldots+\frac{1 \cdot 3 \cdot 5 \cdot(2 n-1) n(n-1) \ldots 1}{n !} D_{0}(x \sqrt{ } 2)\right\}$.

By an easily justified substitution for $D_{2 n}(\sqrt{2} x), D_{2 n-2}(\sqrt{2} x)$ etc. from (19) in (20), the series $(G)$ is obtained.

1 Bull Calcutta Math. Soc., 17, 34. 